\title{
Edge effects on fish associated with seagrass and sand patches
}

\author{
Timothy M. Smith ${ }^{1,2, *}$, Jeremy S. Hindell ${ }^{2,3}$, Gregory P. Jenkins ${ }^{2,3}$, Rod M. Connolly ${ }^{4}$ \\ ${ }^{1}$ Victorian Marine Science Consortium, PO Box 114, Queenscliff, Victoria 3225, Australia \\ ${ }^{2}$ Department of Zoology, The University of Melbourne, Parkville, Victoria 3052, Australia \\ ${ }^{3}$ Marine and Freshwater Systems, Department of Primary Industries Victoria, PO Box 114, Queenscliff, Victoria 3225, Australia \\ ${ }^{4}$ Australian Rivers Institute-Coast and Estuaries, and Griffith School of Environment, Gold Coast Campus, Griffith University, \\ Queensland 4222, Australia
}

\begin{abstract}
Seagrass beds form naturally patchy habitats with large areas of seagrass-sand interface, or edges. Fish were sampled at 3 sites in the temperate waters of Victoria, Australia, using small ( $0.5 \mathrm{~m}$ wide) push nets at 7 positions: unvegetated sand distant from the patch on the seaward side, the sand edge adjacent to the seagrass on the seaward side, the seagrass edge on the seaward side, the middle of the seagrass patch, the seagrass edge on the shore side of the patch, the sand adjacent to the seagrass on the shoreward side of the patch and unvegetated sand distant from the seagrass on the shoreward side. Samples were taken during the day and night, and seagrass variables were collected to describe structural complexity. As expected, more fish were caught in seagrass than over sand. Within seagrass, we found strong and consistent patterns at edges. Regardless of site, the total number of fish sampled was greater at the seaward seagrass edge (484 fish) than in the seagrass middle (231), but there was little difference between the seagrass middle and the shoreward seagrass edge (297). Two species of pipefish, Stigmatopora argus (193) and S. nigra (160), were much more abundant at the seaward seagrass edge than in the seagrass middle at all sites (54 and 46, respectively). The goby Nesogobius maccullochi showed a very different pattern. It was more abundant at the shoreward seagrass edge (127) than over the seagrass middle (31) at all sites, and tended to be more abundant over sand at the edge of seagrass patches than in any other sand positions. The weedfish Cristiceps australis was significantly more abundant at the seaward seagrass edge (26) than in the middle (11), but only at night. Consistent patterns in fish distributions demonstrate clear edge effects both within and alongside seagrass at these sites in south-eastern Australia.
\end{abstract}

KEY WORDS: Seagrass $\cdot$ Fish $\cdot$ Edge effects $\cdot$ Diel cycles $\cdot$ Heterozostera nigricaulis $\cdot$ Unvegetated habitats $\cdot$ Seagrass structure

Resale or republication not permitted without written consent of the publisher

\section{INTRODUCTION}

Landscape attributes play a major role in determining the structure of biological communities (Turner 1989). Proximity, size and within-patch location can influence species diversity, density and interactions within a patch (Fahrig 2003). Edge effects relate to the influence that a patch edge can have in determining species composition and processes within a patch. Edge effects can be considered as, or to influence, patterns in biological and physical parameters such as species richness, predation, food availability, distur- bance, temperature and moisture (Murcia 1995). Although well studied in terrestrial habitats (e.g. review by Fagan et al. 1999), the study of habitat edges in marine environments is in its infancy.

Seagrass is a prominent habitat in nearshore locations worldwide, harbouring a rich and diverse assemblage of fauna (Jackson et al. 2001). Increased fragmentation due to human activity is assumed to be a threat to seagrass fauna (Bostrom et al. 2006). Fragmentation increases the amount of edge associated with seagrass patches and, in conjunction with the propensity of natural seagrass to form patchy mosaics, 
makes it an ideal habitat to study edge effects (Bostrom et al. 2006). Many studies have investigated patch size and assumed that changes in fish assemblages were related to edge effects because the edge-to-area ratio increases with decreasing patch size (reviewed by Bell et al. 2001). However, these studies do not actually demonstrate changes in faunal assemblages across patch edges; instead they show that different sized patches contain different faunal assemblages (Connolly \& Hindell 2006, Jelbart et al. 2006). Few studies have directly assessed edge effects in seagrass habitats.

Studies directly investigating edge effects in seagrass have mostly focused on invertebrates, with fewer studies on fish because of the difficulty in sampling at appropriate spatial scales (Connolly \& Hindell 2006). Studies that have investigated fish patterns at seagrass edges have reported variable responses and, in most cases, no response at all (Connolly \& Hindell 2006). Fish density and species richness often vary little across patch edges (Hovel et al. 2002, Uhrin \& Holmquist 2003, Jelbart et al. 2006), although abundances of individual species may change significantly across patch edges. Jelbart et al. (2006) found that pipefish Urocampus carinirostris were more abundant at the edge of seagrass $(<4 \mathrm{~m}$ from the patch edge) than at the interior (6 to $10 \mathrm{~m}$ from the edge). Conversely, Hovel et al. (2002) found that abundances of pinfish Lagodon rhomboides were lower at seagrass edges, although not at all sampling times. The variability in responses shown by fish to seagrass edges may be, in part, a function of the scale of sampling fish and/or changes in seagrass structure at seagrass edges, causing increases or decreases in fish density.

Fish associated with seagrass have been reported from unvegetated sand adjacent to seagrass patches, which may provide a feeding area in close proximity to shelter (Ferrell \& Bell 1991). Ferrell \& Bell (1991) found that the abundance and diversity of fish over sand within $10 \mathrm{~m}$ of seagrass was less than sand $100 \mathrm{~m}$ from seagrass, and was often more similar to seagrass itself. In general, however, the extent of utilisation of adjacent unvegetated habitats is poorly known. Within seagrass habitat, depth is known to influence fish assemblages (Anderson 2003, Jackson et al. 2006). In shallow marine and estuarine waters where depth increases with distance from shore, it is, therefore, likely that seagrass and sand edges will support different fish assemblages in a shoreward and seaward direction because of the changes in depth.

Variation in fish abundances over diel cycles is well known in seagrass (Edgar \& Shaw 1995) and in other habitats, including coral reefs (Nagelkerken et al. 2000) and unvegetated flats (Gibson et al. 1996). However, edge-related patterns in fish assemblage struc- ture related to diel cycles in seagrass habitats have only recently been investigated (Jackson et al. 2006). Diel changes influence densities of seagrass epifauna (Sanchez-Jerez et al. 1999) and shallow water predators (Gibson et al. 1996) that, in turn, may influence within-patch distributions of fish. Diel variability of fish assemblages across patch edges can therefore be predicted, but needs further study.

In assessing whether fish assemblages vary across patch edges in ways that are consistent with edge effects the present study aims to: (1) quantify how fish assemblages change across seagrass edges, including adjacent sand habitats; (2) determine whether withinpatch variability in fish assemblage structure changes with diel periods, patch edge location and water depth; and (3) determine the extent to which observed patterns vary spatially (among sites).

\section{METHODS}

Study sites. Sampling was done in Port Phillip Bay, between October 2005 and January 2006. Port Phillip Bay is a large, semi-enclosed bay that is predominantly marine, with a small $(1 \mathrm{~m})$ tidal range. Three sites were chosen within Port Phillip Bay: Blairgowrie in the southeast $\left(38^{\circ} 21^{\prime} 46^{\prime \prime} \mathrm{S}, 144^{\circ} 47^{\prime} 21^{\prime \prime} \mathrm{E}\right.$, Site 1$)$, and Grassy Point ( $38^{\circ} 07^{\prime} 15^{\prime \prime} \mathrm{S}, 144^{\circ} 41^{\prime} 38^{\prime \prime} \mathrm{E}$, Site 2$)$ and Indented Head ( $38^{\circ} 09^{\prime} 32^{\prime \prime} \mathrm{S}, 144^{\circ} 43^{\prime} 12^{\prime \prime} \mathrm{E}$, Site 3$)$ on the central-western coast of the bay (Fig. 1). Samples were taken over a $16 \mathrm{wk}$ period in which abundances

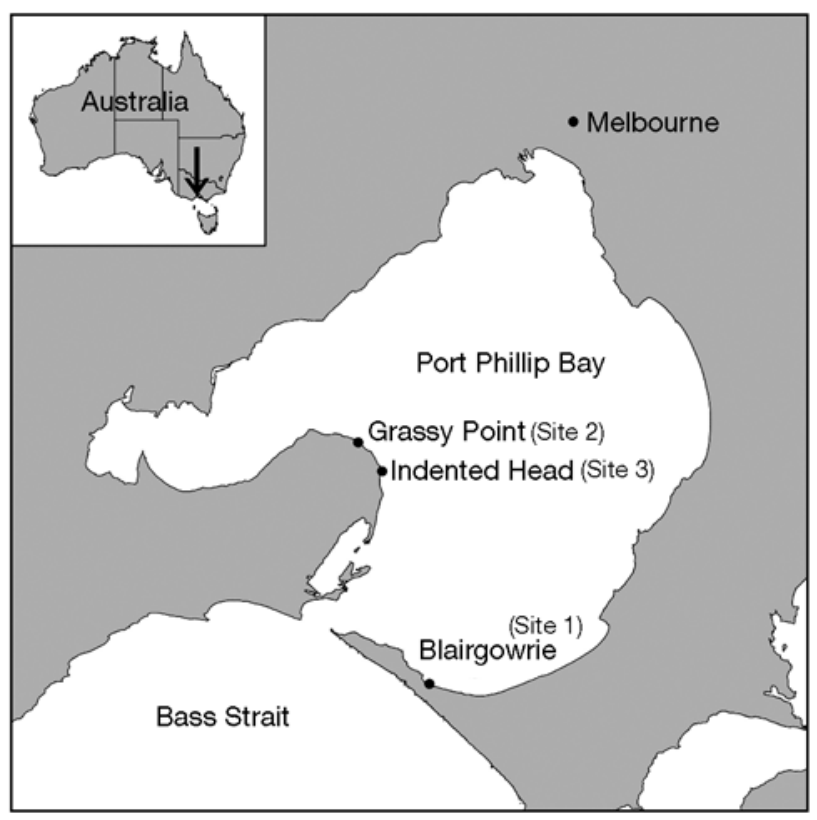

Fig. 1. Location of study sites in Port Phillip Bay. Insert: Location of Port Phillip Bay within Australia 
of fish could be influenced by ontogeny, behaviour and turnover. Such temporal variation between replicates adds rigour to our results and increases the generality of our findings.

Sites are protected from the predominant southwesterly winds and support patches of the seagrass Heterozostera nigricaulis, running parallel to the shore at depths of $<1.5 \mathrm{~m}$, interspersed by $10 \mathrm{~s}$ of metres of unvegetated sand.

At each site, 4 seagrass patches were chosen for sampling that were $>50 \mathrm{~m}$ apart, $>10 \mathrm{~m}$ in diameter and showed distinct seagrass/sand boundaries. Patches ranged in size, shape and seagrass structure (Table 1).

Sampling. Fish: Fish were sampled in the 4 patches at the 3 sites during the day and night to assess fish patterns and diel periods. Seven positions were sampled at each patch (Fig. 2):

(1) The unvegetated sand in the seaward region at the equivalent distance from the seagrass patch edge, as the middle of the seagrass patch is to the seagrass edge (UV Sea).

(2) Unvegetated sand within $1 \mathrm{~m}$ of the seagrasssand interface in the seaward region (UV-E Sea).

(3) Seagrass within $1 \mathrm{~m}$ of the seagrass-sand interface in the seaward region (SG-E Sea).

(4) The seagrass middle (SG Mid).

(5) Seagrass within $1 \mathrm{~m}$ of the seagrass-sand interface in the shoreward region (SG-E Shore).

(6) Unvegetated sand within $1 \mathrm{~m}$ of the seagrasssand interface in the shoreward region (UV-E Shore).

(7) Unvegetated sand in the shoreward region at the equivalent distance from the patch edge, as the middle of the seagrass patch is to the seagrass edge (UV Shore).

Table 1. Heterozostera nigricaulis. Area and average (SE in parentheses) seagrass variables in each patch at each site

\begin{tabular}{|c|c|c|c|c|c|}
\hline Patch & $\begin{array}{l}\text { Area } \\
\left(\mathrm{m}^{-2}\right)\end{array}$ & $\begin{array}{l}\text { Biomass } \\
\left(\mathrm{g} \mathrm{m}^{-2}\right)\end{array}$ & $\begin{array}{l}\text { Shoot density } \\
\qquad\left(\mathrm{m}^{-2}\right)\end{array}$ & $\begin{array}{l}\text { Length } \\
(\mathrm{mm})\end{array}$ & $\begin{array}{l}\text { Epiphyte biomass } \\
\qquad\left(\mathrm{g} \mathrm{m}^{-2}\right)\end{array}$ \\
\hline \multicolumn{6}{|l|}{ Site 1} \\
\hline 1 & 3098 & 174 (11) & 11980 (625) & 151 & 28 (1.9) \\
\hline 2 & 461 & $66(2)$ & 8341 (215) & $85 i<0.1$ & $4 \quad(0.4)$ \\
\hline 3 & 269 & 141 (4) & 10643 (80) & 145 (2) & $13(0.8)$ \\
\hline 4 & 5934 & 125 (6) & $9793(240)$ & $112(2)$ & $3(0.4)$ \\
\hline \multicolumn{6}{|l|}{ Site 2} \\
\hline 1 & 2851 & 165 (8) & 21561 (937) & 141 & $3(0.2)$ \\
\hline 2 & 5297 & 139 (9) & 18453 (857) & 106 & $7(0.8)$ \\
\hline 3 & 2740 & 139 (5) & 15931 (430) & 133 (3) & 41 (3.0) \\
\hline 4 & 747 & 224 (1) & 20333 (429) & 195 & $11(0.9)$ \\
\hline \multicolumn{6}{|l|}{ Site 3} \\
\hline 1 & 2178 & 177 (17) & 17637 & $128(7)$ & $12(1.6)$ \\
\hline 2 & 402 & $59(4)$ & $9436(472)$ & 79 (2) & $12(1.0)$ \\
\hline 3 & 2238 & 131 (8) & 16680 (593) & 112 & $35(2.5)$ \\
\hline 4 & 11518 & 112 (3) & 14947 (170) & 120 & 48 (3.4) \\
\hline
\end{tabular}

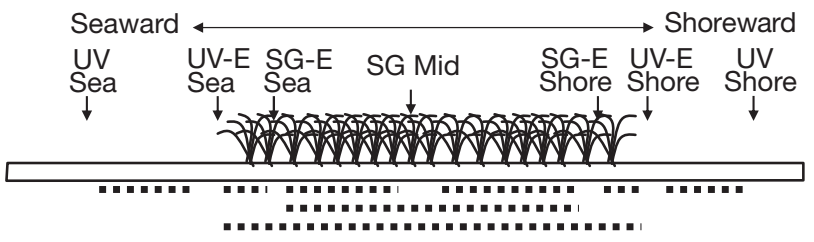

Fig. 2. Positions sampled within each seagrass Heterozostera nigricaulis (SG) patch. At each patch SG Mid was equal to half the distance between SG-E Sea and SG-E Shore, which was the same distance unvegetated (UV) Sea and Shore were from the seagrass edge. Dotted lines represent all planned comparisons, except between seagrass and unvegetated positions. For full description of site/patch abbreviations see 'Methods; Sampling; Fish'

Sampling was done on 8 occasions (4 days and 4 nights) for each patch at each site. Fish samples were collected at each position on each sampling occasion. Fish were sampled using a $1 \times 0.5 \mathrm{~m}$ push net with $1 \mathrm{~mm}$ mesh, pushed parallel to the patch edge for $5 \mathrm{~m}$. Sampled fish were anaesthetised and preserved in ethanol for later identification and counting.

Seagrass structure and depth: Seagrass structure can change across patches and affect fish assemblages (Anderson 2003), potentially confounding the influence of an edge. To document changes in seagrass structure (biomass, length, shoot density, epiphyte biomass) across positions, 4 haphazardly placed samples of seagrass were collected at the completion of the faunal sampling at each seagrass position within each patch at each site $(4$ samples $\times 3$ positions $\times 4$ patches $\times$ 3 sites = 144 samples). Seagrass samples were taken by cutting all seagrass within a $0.25 \mathrm{~m}^{2}$ quadrat. Water depth was also measured at the point of each fish sample. In the laboratory, macroalgae in the seagrasssamples were separated from seagrass. The numbers of shoots and length of leaves were measured for seagrass. Seagrass and algae were weighed after drying to constant weight at $60^{\circ} \mathrm{C}$.

Data analysis. Data were assessed for assumptions of homogeneity of variance and normality by viewing boxplots and plots of residuals (Quinn $\&$ Keough 2002). Where these assumptions were not met, samples were log transformed and reassessed (Quinn \& Keough 2002).

Fish: Total fish densities, species richness and densities of the most abundant fish species were analysed using 4-factor, nested analyses of variance (ANOVA). Position (7 levels), site (3 levels) and time of day (2 levels) 
were treated as fixed factors, and seagrass patches (4 in each site) were nested within sites. UV Shore positions were not taken at Patches 1 and 4 at Site 1, because these patches were too close to the shore and other suitable patches could not be found. We were not interested in small-scale temporal variation; therefore, we averaged fish samples across sampling occasions for each patch to simplify the ANOVA model. Specific planned comparisons were run (Fig. 2), and Tukey's tests used to determine differences among sites.

Table 2. Heterozostera nigricaulis. ANOVA results for seagrass biomass, length, shoot density and epiphyte biomass. Significant results in bold $(\mathrm{p}<0.05)$

\begin{tabular}{|c|c|c|c|c|c|c|c|c|c|}
\hline \multirow{2}{*}{ Source } & \multirow{2}{*}{$\mathrm{df}$} & \multicolumn{2}{|c|}{ Seagrass biomass } & \multicolumn{2}{|c|}{ Blade length } & \multicolumn{2}{|c|}{ Shoot density } & \multirow{2}{*}{$\begin{array}{c}\text { Epiphyte } \\
F\end{array}$} & biomass \\
\hline & & $F$ & $\mathrm{p}$ & $F$ & $\mathrm{p}$ & $F$ & $\mathrm{p}$ & & $\mathrm{p}$ \\
\hline Site & 2 & 2.8 & 0.111 & 1.8 & 0.228 & 9.6 & 0.006 & 2.8 & 0.596 \\
\hline Position & 2 & 27.6 & $<0.001$ & 35.3 & $<0.001$ & 11.5 & 0.001 & 27.6 & 0.004 \\
\hline Position $\times$ Site & 4 & 4.7 & 0.009 & 4.4 & 0.012 & 2.2 & 0.112 & 4.7 & 0.276 \\
\hline Patch(Site) & 9 & 6.5 & $<0.001$ & 13.8 & $<0.001$ & 2.6 & 0.010 & 6.5 & $<0.001$ \\
\hline Position $\times$ Patch $($ Site $)$ & 18 & 2.7 & 0.001 & 2.7 & 0.001 & 2.8 & 0.001 & 2.7 & $<0.001$ \\
\hline Error & 108 & & & & & & & & \\
\hline
\end{tabular}
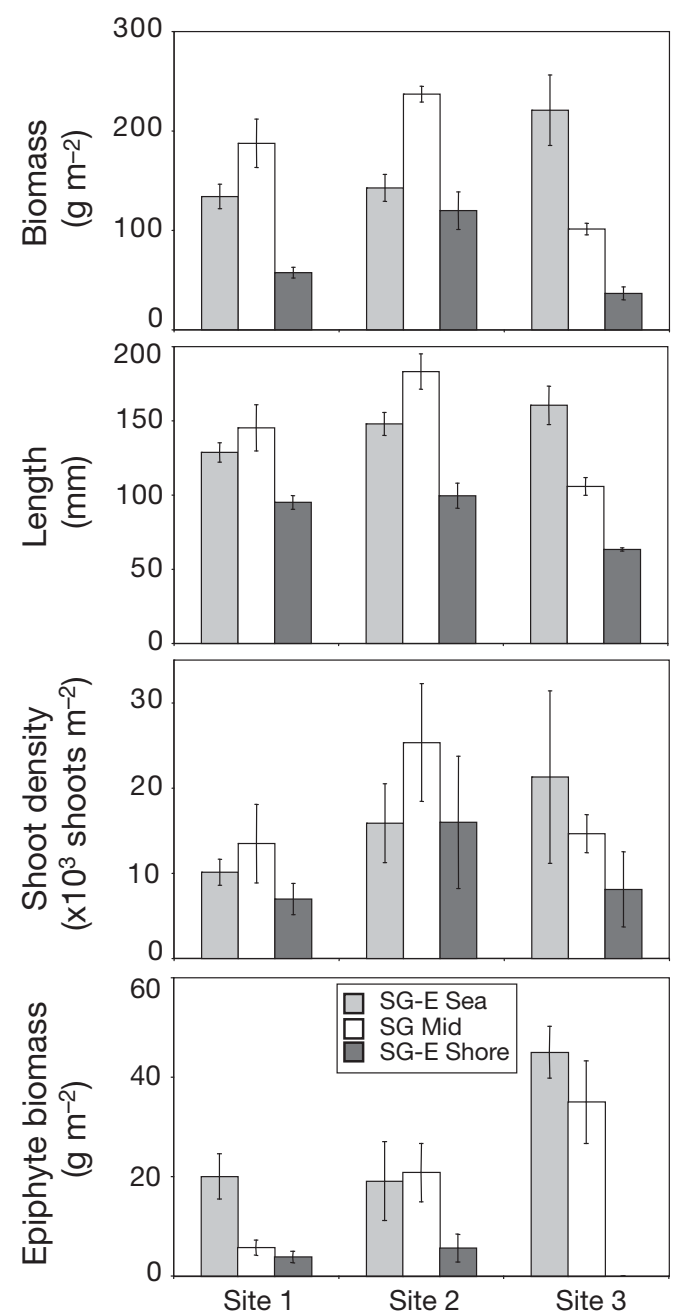

Fig. 3. Heterozostera nigricaulis. Average seagrass biomass, length, shoot density and epiphyte biomass at each site and position $( \pm 1 \mathrm{SE})$
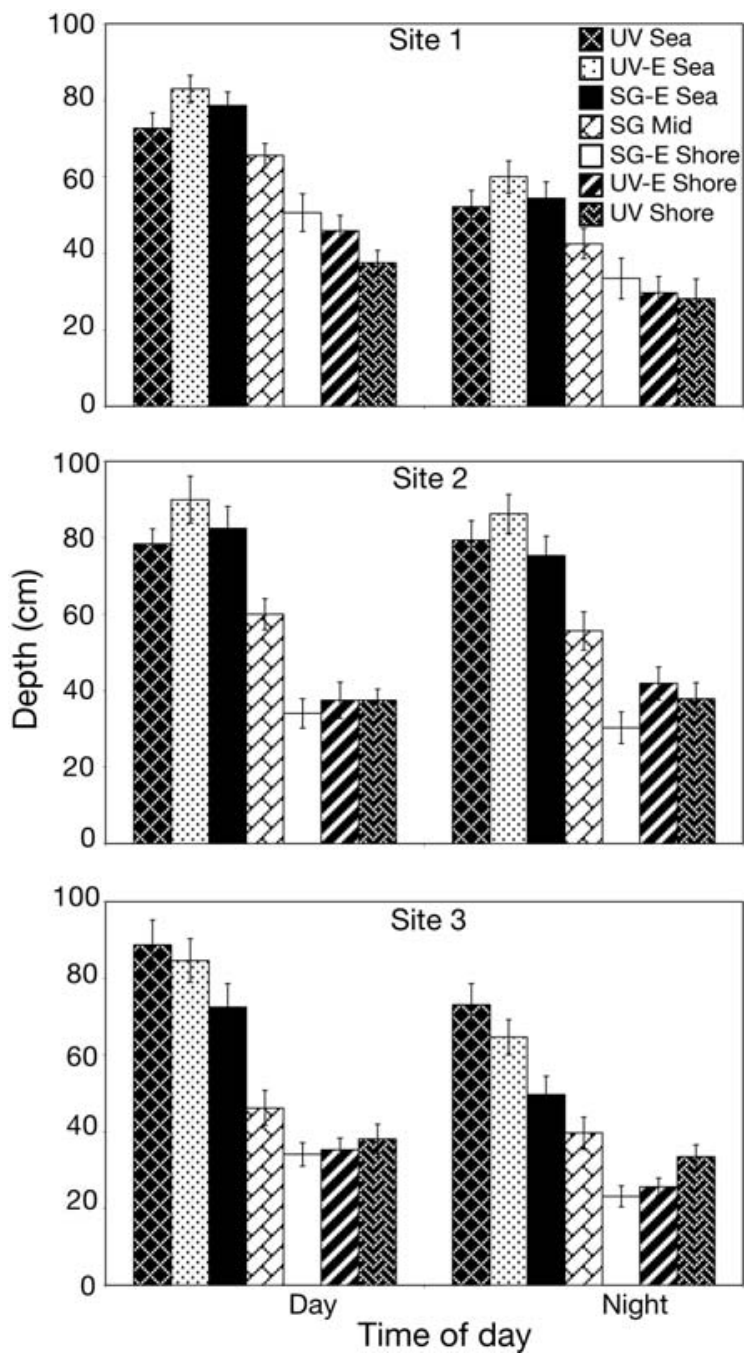

Fig. 4. Average depth at each position within each site $( \pm 1 \mathrm{SE})$ 
Seagrass structure and depth: Variation in seagrass structure, including seagrass biomass, epiphyte biomass, seagrass length and seagrass shoot density across positions, was examined using 3-factor ANOVAs; site and position were fixed factors, while patches were nested within sites.

A 4-factor ANOVA was used to determine if depth varied across positions, sites and diel periods. Position, site and time of day were treated as fixed factors, while patches were nested within site. Specific planned comparisons were used to assess differences between positions (as for fish densities), and Tukey's tests were used to differentiate among sites.

\section{RESULTS}

\section{Seagrass structure and depth}

Seagrass Heterozostera nigricaulis biomass and length varied among positions, but with a different pattern at different sites (Table 2). Seagrass was generally longer and more dense and therefore had greater biomass at SG-E Sea and SG Mid at Site 1, at the Mid position at Site 2, and at SG-E Sea at Site 3. SG-E
Shore always had the lowest seagrass variables (Fig. 3).

There were significant differences in depth among positions that varied with site $\left(F_{12,113}=3.6, \mathrm{p}<0.001\right)$, and between times of day that also varied with site $\left(F_{2,113}=10.7, \mathrm{p}<0.001\right)$. At all sites and times of the day, however, depth was greater at the seaward positions than in the middle, which was deeper than the shoreward positions (Fig. 4). Depth at each patch within each site also varied $\left(F_{9,113}=10.5, \mathrm{p}<0.001\right)$.

\section{Fish}

A total of 2094 fish, from 24 species and 12 families was caught. Larval Atherinidae dominated samples numerically (689 individuals); however, atherinids are highly mobile, transient schooling fish that are not seagrass residents and were therefore removed from further analyses. Stigmatopora nigra was the second most abundant species (377 individuals), followed by $\mathrm{S}$. argus (376), $\mathrm{Ne}$ sogobius maccullochi (295), Heteroclinus adelaide (125), Sillaginodes punctata (59) and Cristiceps australis (57); these 6 species represented $92 \%$ of the fish sampled (Table 3) and represent seagrass canopy (S. nigra, S. ar-

Table 3. Total abundances of fish sampled at each position at all sites and times. For full description of site/patch abbreviations see 'Methods; Sampling; Fish'

\begin{tabular}{|c|c|c|c|c|c|c|c|c|c|c|}
\hline Family & Common name & Species & $\begin{array}{l}\text { UV } \\
\text { Sea }\end{array}$ & $\begin{array}{c}\text { UV-E } \\
\text { Sea }\end{array}$ & $\begin{array}{c}\text { SG-E } \\
\text { Sea }\end{array}$ & $\begin{array}{l}\text { SG } \\
\text { Mid }\end{array}$ & $\begin{array}{l}\text { SG-E } \\
\text { Shore }\end{array}$ & $\begin{array}{l}\text { UV-E } \\
\text { Shore }\end{array}$ & $\begin{array}{l}\text { UV } \\
\text { Shore }\end{array}$ & Total \\
\hline \multirow[t]{3}{*}{ Atherinidae } & & Atherinosoma sp. & 0 & 0 & 0 & 0 & 0 & 0 & 1 & 1 \\
\hline & Pikehead hardyhead & Kestratherina esox & 0 & 2 & 2 & 0 & 0 & 1 & 0 & 5 \\
\hline & Silver fish & Leptatherina presbyteroides & 0 & 0 & 0 & 0 & 1 & 0 & 0 & 1 \\
\hline \multirow[t]{3}{*}{ Clinidae } & Southern crested weedfish & Cristiceps australis & 0 & 8 & 26 & 11 & 8 & 4 & 0 & 57 \\
\hline & Adelaide weedfish & Heteroclinus adelaide & 0 & 3 & 52 & 52 & 16 & 2 & 0 & 125 \\
\hline & Weedfish larvae & & 1 & 0 & 0 & 0 & 0 & 0 & 0 & 1 \\
\hline Enoplosidae & Old wife & Enoplosus armatus & 0 & 0 & 2 & 0 & 0 & 0 & 0 & 2 \\
\hline \multirow[t]{2}{*}{ Gobiidae } & Bridled goby & Arenigobius bifrentatus & 0 & 3 & 0 & 0 & 1 & 1 & 0 & 5 \\
\hline & Goby & Nesogobius maccullochi & 8 & 33 & 12 & 31 & 127 & 72 & 12 & 295 \\
\hline \multirow[t]{2}{*}{ Monacanthidae } & Leatherjacket & Acanthaluteres sp. & 1 & 4 & 15 & 14 & 13 & 1 & 0 & 48 \\
\hline & Six spine leatherjacket & Meuschenia freycineti & 0 & 0 & 3 & 0 & 0 & 0 & 0 & 3 \\
\hline Odacidae & Little rock whiting & Neodax balteatus & 0 & 0 & 1 & 0 & 0 & 0 & 0 & 1 \\
\hline Pleuronectidae & Greenback flounder & Rhombosolea tapirina & 0 & 0 & 0 & 0 & 5 & 0 & 2 & 7 \\
\hline Scorpaenidae & Cobbler & Gymnapistes marmoratus & 3 & 5 & 2 & 6 & 9 & 3 & 1 & 29 \\
\hline Scorpidiae & Moonlighter & Tilodon sexfasciatum & 0 & 0 & 0 & 1 & 0 & 0 & 0 & 1 \\
\hline Sillaginidae & King George whiting & Sillaginodes punctata & 0 & 8 & 12 & 14 & 22 & 1 & 2 & 59 \\
\hline \multirow[t]{5}{*}{ Syngnathidae } & Spotted pipefish & Stigmatopora argus & 1 & 53 & 193 & 54 & 61 & 13 & 1 & 376 \\
\hline & Wide body pipefish & Stigmatopora nigra & 5 & 108 & 160 & 46 & 33 & 23 & 2 & 377 \\
\hline & Halfbanded pipefish & Mitotichthys semistriatus & 0 & 0 & 1 & 1 & 0 & 0 & 0 & 2 \\
\hline & Pugnose pipefish & Pugnaso curtirostris & 2 & 1 & 2 & 0 & 0 & 0 & 0 & 5 \\
\hline & Port Phillip pipefish & Vanacampus phillipi & 0 & 1 & 0 & 1 & 1 & 0 & 0 & 3 \\
\hline \multirow[t]{2}{*}{ Tetraodontidae } & & Contusus sp. & 0 & 0 & 1 & 0 & 0 & 0 & 0 & 1 \\
\hline & Smooth toadfish & Tetractenos glaber & 0 & 1 & 0 & 0 & 0 & 0 & 0 & 1 \\
\hline Total & & & 21 & 230 & 484 & 231 & 297 & 121 & 21 & 1405 \\
\hline
\end{tabular}


Table 4. ANOVA results for fish density and species richness. Significant results in bold $(\mathrm{p}<0.05)$. TOD: time of day

\begin{tabular}{|c|c|c|c|c|c|}
\hline \multirow[t]{2}{*}{ Source } & \multirow[t]{2}{*}{$\mathrm{df}$} & \multicolumn{2}{|c|}{ Fish density } & \multicolumn{2}{|c|}{ Species richness } \\
\hline & & $F$ & $\mathrm{p}$ & $F$ & $\mathrm{p}$ \\
\hline Site & 2 & 2.1 & 0.185 & 3.4 & 0.078 \\
\hline Position & 6 & 26.5 & $<0.001$ & 36.1 & $<0.001$ \\
\hline TOD & 1 & 14.3 & $<0.001$ & 58.0 & $<0.001$ \\
\hline Position $\times$ Site & 12 & 3.0 & 0.001 & 3.4 & $<0.001$ \\
\hline Position $\times$ TOD & 6 & 1.2 & 0.319 & 1.0 & 0.417 \\
\hline Site $\times$ TOD & 2 & 5.6 & 0.005 & 0.9 & 0.435 \\
\hline Site $\times$ Position $\times$ TOD & 12 & 0.8 & 0.624 & 0.4 & 0.965 \\
\hline Patch(Site) & 9 & 3.7 & $<0.001$ & 3.8 & $<0.001$ \\
\hline Error & 113 & & & & \\
\hline
\end{tabular}

gus, S. punctata), sand (N. maccullochi) and benthic (H. adelaide, C. australis) species. Seven species were sampled exclusively in seagrass, while only 3 were sampled only on unvegetated sand, 14 species were sampled over both habitats, including the 6 most abundant species (Table 3). Four species (S. nigra, S. argus, N. maccullochi and $C$. australis) showed a strong response to seagrass edges when analysed separately. The vast majority of N. maccullochi (63\%), S. punctata (84\%), Acanthaluteres sp. (92\%) and Gymnapistes marmoratus (90\%) were caught at Site 1. Overall catch per unit effort (CPUE) was low, but, due to the sampling technique required to sample fish on a fine spatial scale and the large portion of samples taken on unvegetated habitats, low CPUE was not unexpected.
Fish density

Fish density varied for seagrass position and time of day, but both patterns were inconsistent amongst sites (Table 4). At Sites 2 and 3, density was greater at UVE Sea than at UV Sea, and, at Site 1, UV-E Shore was greater than UV Shore (Table 5, Fig. 5). When each site was considered individually, fish density was always greater at SG-E Sea than at SG Mid, but there was no difference between SG-E Shore and SG Mid (Table 5, Fig. 5).

\section{Species richness}

Species richness differed among positions, but not in the same way at each site (Table 4). Within-patch

Table 5. Planned comparison results for fish density and species richness at each site. ${ }^{*} \mathrm{p}<0.05 ;{ }^{* *} \mathrm{p}<0.01 ;{ }^{* * *} \mathrm{p}<0.001$

\begin{tabular}{|c|c|c|c|c|c|c|}
\hline & \multicolumn{3}{|c|}{ Density } & \multicolumn{3}{|c|}{ Species richness } \\
\hline & Site 1 & Site 2 & Site 3 & Site 1 & Site 2 & Site 3 \\
\hline Vegetated vs. unvegetated & $* * *$ & $* * *$ & & $* * *$ & $* * *$ & $* * *$ \\
\hline Seagrass & & & & & & \\
\hline SG-E Sea vs. SG Mid & * & ** & * & & & ** \\
\hline SG-E Shore vs. SG Mid & & & & *** & & \\
\hline SG-E Sea vs. SG-E Shore & & $* *$ & & $* * *$ & & \\
\hline Unvegetated & & & & & & \\
\hline UV-E Sea vs. UV Sea & & *** & $* * *$ & $* * *$ & $* * *$ & $* * *$ \\
\hline UV-E Shore vs. UV Shore & * & & & $* * *$ & & \\
\hline Sand Sea vs. Sand Shore & & ${ }^{* *}$ & *** & $* * *$ & *** & $* * *$ \\
\hline Mixed & & & & & & \\
\hline SG-E Sea vs. UV-E Sea & *** & * & & *** & & \\
\hline SG-E Shore vs. UV-E Shore & ** & * & & **** & *** & * \\
\hline
\end{tabular}
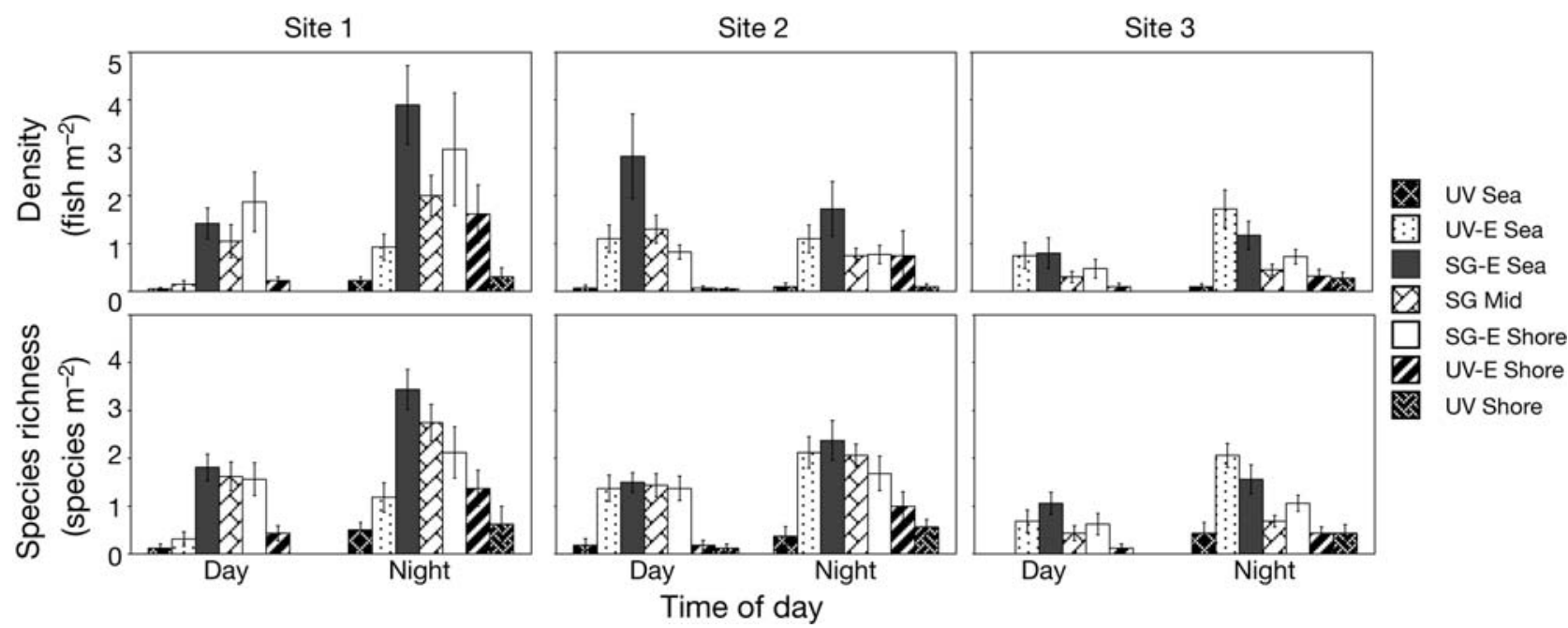

Time of day

Fig. 5. Fish density and species richness within each position, at each site during the day and night $( \pm 1 \mathrm{SE})$ 
differences were only found at Site 1 (SG Mid > SG-E Shore) and Site 3 (SG-E Sea > SG Mid) (Table 5, Fig. 5). Although species richness was greater in the seagrass than in the unvegetated positions and greater at UV-E Sea than at the more distant UV Sea for all sites, at Sites 2 and 3 there was no difference in richness between SG-E Sea and UV-E Sea (Table 5, Fig. 5).

\section{Stigmatopora nigra}

Stigmatopora nigra densities differed among positions, but patterns varied across sites (Table 6). When each site was considered individually, S. nigra densities were greater at the seaward edge than in the middle of seagrass patches at all sites, but there was no difference between the shoreward edge and middle (Table 7, Fig. 6). S. nigra used unvegetated edge positions extensively at Site 2, where densities were greater at UV-E Sea than at UV Sea, and, at Site 3, where densities were greater at UV-E Sea than at SGE Sea (Table 7, Fig. 6).

\section{Stigmatopora argus}

Stigmatopora argus densities also differed among positions, but inconsistently across sites (Table 6). At Sites 1 and 2, S. argus densities were greater at SGE Sea than at SG Mid, and there was no difference between SG-E Shore and SG Mid (Table 7, Fig. 6). However, these patterns were not apparent at Site 3, where few $S$. argus were sampled. $S$. argus were not sampled in unvegetated positions at Site 1, but, at Site 2, densities were greater at UV-E Sea than at UV Sea (Table 7, Fig. 6).

\section{Nesogobius maccullochi}

Densities of the goby Nesogobius maccullochi varied with site and differed among positions, but in a different way during the day and night (Table 6). During both the day and night, more $N$. maccullochi were sampled at the shoreward seagrass edge than in the middle. At night, N. maccullochi were always sampled in greater densities at the unvegetated edge positions than in the more distant unvegetated positions

Table 6. ANOVA results for individual fish species. Significant results in bold $(\mathrm{p}<0.05)$. TOD: time of day

\begin{tabular}{|c|c|c|c|c|c|c|c|c|c|}
\hline \multirow[t]{2}{*}{ Source } & \multirow[t]{2}{*}{$\mathrm{df}$} & \multicolumn{2}{|c|}{$\begin{array}{c}\text { Stigmatopora } \\
\text { nigra }\end{array}$} & \multicolumn{2}{|c|}{$\begin{array}{c}\text { Stigmatopora } \\
\text { argus }\end{array}$} & \multicolumn{2}{|c|}{$\begin{array}{l}\text { Nesogobius } \\
\text { maccullochi }\end{array}$} & \multicolumn{2}{|c|}{$\begin{array}{l}\text { Cristiceps } \\
\text { australis }\end{array}$} \\
\hline & & $F$ & $\mathrm{p}$ & $F$ & $\mathrm{p}$ & $F$ & $\mathrm{p}$ & $F$ & $\mathrm{p}$ \\
\hline Site & 2 & 0.3 & 0.718 & 4.9 & 0.036 & 9.6 & 0.006 & 4.6 & 0.042 \\
\hline Position & 6 & 17.4 & $<0.001$ & 13.7 & $<0.001$ & 13.1 & $<0.001$ & 4.7 & $<0.001$ \\
\hline TOD & 1 & 0.4 & 0.512 & $<0.1$ & 0.962 & 20.1 & $<0.001$ & 16.8 & $<0.001$ \\
\hline Position $\times$ Site & 12 & 4.0 & $<0.001$ & 3.6 & $<0.001$ & 1.3 & 0.231 & 1.7 & 0.075 \\
\hline Position $\times$ TOD & 6 & 1.7 & 0.123 & 0.3 & 0.912 & 2.3 & 0.037 & 3.3 & 0.005 \\
\hline Site $\times$ TOD & 2 & 1.6 & 0.203 & 1.5 & 0.237 & 2.9 & 0.058 & 1.3 & 0.272 \\
\hline Site $\times$ Position $\times$ TOD & 12 & 1.5 & 0.130 & 0.7 & 0.706 & 1.0 & 0.483 & 1.4 & 0.164 \\
\hline Patch(Site) & 9 & 3.6 & 0.001 & 2.9 & 0.004 & 1.6 & 0.114 & 1.1 & 0.360 \\
\hline Error & 113 & & & & & & & & \\
\hline
\end{tabular}

Table 7. Planned comparison results for individual fish species within each site and time of day. ${ }^{*} p<0.05 ;{ }^{* * *} p<0.01 ;{ }^{* * *} p<0.001$; -: insufficient fish sampled to perform analysis

\begin{tabular}{|c|c|c|c|c|c|c|c|c|c|c|}
\hline & \multicolumn{3}{|c|}{$\begin{array}{c}\text { Stigmatopora } \\
\text { nigra }\end{array}$} & \multicolumn{3}{|c|}{$\begin{array}{c}\text { Stigmatopora } \\
\text { argus }\end{array}$} & \multicolumn{2}{|c|}{$\begin{array}{l}\text { Nesogobius } \\
\text { maccullochi }\end{array}$} & \multicolumn{2}{|c|}{$\begin{array}{c}\text { Cristiceps } \\
\text { australis }\end{array}$} \\
\hline & Site 1 & Site 2 & Site 3 & Site 1 & Site 2 & Site 3 & Day & Night & Day & Night \\
\hline Vegetated vs. Unvegetated & $* * *$ & $* * *$ & $* * *$ & $* * *$ & $* * *$ & $* * *$ & $* * *$ & $* * *$ & $* * *$ & $* * *$ \\
\hline \multicolumn{11}{|l|}{ Seagrass } \\
\hline SG-E Sea vs. SG Mid & $* *$ & $*$ & $* * *$ & $* * *$ & ** & & & & & $* * *$ \\
\hline SG-E Shore vs. SG Mid & & & & & & & $* * *$ & $* * *$ & & \\
\hline SG-E Sea vs. SG-E Shore & $* * *$ & $* *$ & $* *$ & ${ }^{* *}$ & $* * *$ & & $* * *$ & $* * *$ & & $* * *$ \\
\hline \multicolumn{11}{|l|}{ Unvegetated } \\
\hline UV-E Sea vs. UV Sea & & $* *$ & $* * *$ & - & $* *$ & & & $*$ & & \\
\hline UV-E Shore vs. UV Shore & & & & - & & & & $* *$ & & \\
\hline UV-E Sea vs. UV-E Shore & & * & $* * *$ & - & * & & & * & & \\
\hline \multicolumn{11}{|l|}{ Mixed } \\
\hline SG-E Sea vs. UV-E Sea & $* * *$ & & $*$ & $* * *$ & $* *$ & & & * & & \\
\hline SG-E Shore vs. UV-E Shore & & & & ** & & & $* * *$ & & & \\
\hline
\end{tabular}


(Table 7, Fig. 6). During the day, more N. maccullochi were sampled at SG-E Shore than at UV-E Shore, but there was no difference during the night.

\section{Cristiceps australis}

The weedfish Cristiceps australis was sampled in greater densities at SG-E Sea than at both SG Mid and SG-E Shore during the night, but not during the day (Tables 6 \& 7, Fig. 6).

\section{Relationships between fish densities and seagrass structure}

Fish density, both overall and of individual species, showed different patterns to those of seagrass structure. For example, at Site 1, there was no difference in seagrass biomass at SG-E Sea and at SG Mid, but there were clearly fewer fish in the patch middle (Figs. 3 \& 5). Similarly, at Site 2, seagrass biomass was greatest in the middle, but Stigmatopora nigra densities were much greater at the seaward edge (Figs. $3 \& 6$ ).

\section{DISCUSSION}

Fish assemblages can vary according to within-patch location, but the presence of an edge effect in seagrass habitats has been inconclusive. While Uhrin \& Holmquist (2003) found fish densities to be greatest $10 \mathrm{~m}$ from the seagrass edge, and others found changes in individual species densities at the seagrass edge (Hovel et al. 2002, Jelbart et al. 2006), most studies have found no difference in fish density between the edge and interior (Connolly \& Hindell 2006). We

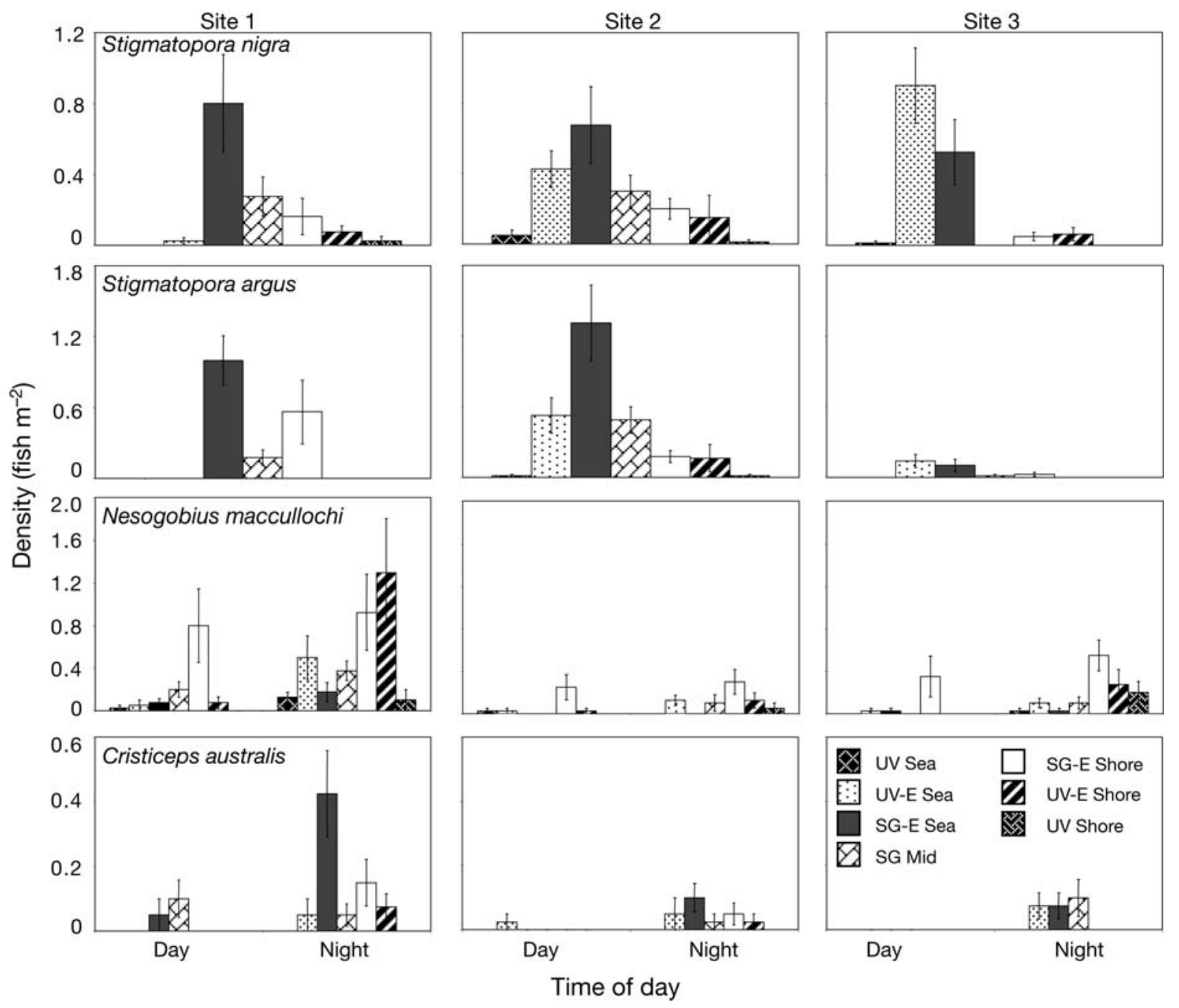

Fig. 6. Average density at each site of Stigmatopora nigra, S. argus, Nesogobius maccullochi and Cristiceps australis at each position during each diel period $( \pm 1 \mathrm{SE})$ 
found both overall and individual fish densities were greater at seagrass edges than in the middle. Fish density was greater at the seaward edge than in the middle, but not between the shoreward edge and middle. Similarly, the pipefish Stigmatopora nigra and S. argus and the weedfish Cristiceps australis were much more common at the seaward edge than in the seagrass middle, although $C$. australis only showed this pattern during the night. The goby Nesogobius maccullochi, by contrast, was more abundant at the edges in the shoreward direction than in the seagrass middle. Patterns of both overall and individual within-patch fish distributions provide support for edge effects, contrasting with previous work that has found little evidence of seagrass edge effects (Connolly \& Hindell 2006).

Seagrass edges may provide pipefish with an advantage over other microhabitats within a seagrass patch. Pipefish feed on planktonic copepods and other small crustaceans (Kendrick \& Hyndes 2005), which may be more plentiful at the seagrass edge (Tanner 2005), suggesting that pipefish may use the edge because there is greater food availability.

Unvegetated habitats support fewer fishes than seagrass habitats (Connolly 1994), but use of sand at the seagrass-sand interface by seagrass-associated fish is not well known. Anderson (2003) found that sandassociated fishes such as flatheads and stingarees were more common in close proximity to structure than in completely unvegetated habitats, supporting the findings of Ferrell \& Bell (1991) that non-seagrass fishes are more abundant in sand within $10 \mathrm{~m}$ of seagrass than in either seagrass, or sand $>100 \mathrm{~m}$ away. We found that sand habitats immediately adjacent to seagrass patches were used extensively by fish, particularly in relation to more distant unvegetated habitats. Fish density and number of species were greater over sand at the edge of seagrass than over the more distant unvegetated sand, and the 6 most abundant species were all sampled at the sand edge. Additionally, seagrass-associated shrimp species sampled in the present study, while showing no difference in distribution with seagrass patches, were sampled in greater densities in adjacent sand than in distant sand (T. M. Smith unpubl. data). At Site 1, however, few fish were sampled at the sand edge. Fish prey items (meiofaunal crustaceans) are lower in abundance (Jenkins \& Hamer 2001) and predation pressure greater (Hindell et al. 2002) in unvegetated habitats at Site 1 than at other seagrass-associated sites within Port Phillip Bay, suggesting that predation or food availability reduce fish utilisation of the sand edges at this site. Sand at the seagrass edge may provide a place for fish to forage while still within close proximity to the safety of seagrass, which more distant sand habitats cannot provide (Anderson 2003); however, more experimental work is needed to determine the importance of unvegetated habitats directly next to seagrass patches.

Fish assemblages can change with diel cycles (Nagelkerken et al. 2000), but within-patch diel variations have only recently been investigated. Jackson et al. (2006) found that the influence of seagrass landscape attributes, including edge effects, on fish assemblages change with diel cycles. In the present study, although more fish were sampled at night, time of day had little effect on fish microhabitat choice for most species. The one exception was Cristiceps australis, which was more abundant at the seaward seagrass edge than at other positions at night, but not during the day. Movement to the seagrass edge at night may reflect changes in predator abundance or food availability, but too little is known of the ecology of C. australis to draw firm conclusions for the species.

Changes in seagrass structure within seagrass patches can influence fish assemblages (Bologna \& Heck 2002, Jelbart et al. 2007), potentially explaining edge effects within seagrass habitats. The response of fish in relation to seagrass structure (biomass, length, density, epiphytes) has been well studied (Anderson 2003, Hyndes et al. 2003, Jackson et al. 2006), increasing or decreasing fish density depending on species. We found that seagrass structure varied across and within patches, possibly explaining differences in fish assemblages within patches. Differences in structure between the seaward edge and middle varied inconsistently across sites. Fish densities between the seaward edge and middle, however, were consistent across sites, suggesting that within-patch differences in structure do not determine fish assemblages. Although depth can influence the distribution of a variety of fishes (Jackson et al. 2006), the depth at the seaward edge and middle was always much greater than at the seagrass canopy, and was unlikely to influence the small seagrass fish sampled in the present study. The shoreward seagrass edge always had lower seagrass density, biomass and length and was shallower than the middle of a patch, and therefore might be unfavourable for some species (Jackson et al. 2006), potentially masking any edge effect at the shoreward edge. Conversely, Nesogobius maccullochi showed a preference for the shoreward edge, which may reflect a preference for sparse, short seagrass and shallow water, and not an edge effect. Ries \& Sisk (2004) emphasised that variations in patch quality will cause variability in species responses to edges. We found that fish showed a strong response to the seaward seagrass edge, but little to the shoreward edge. Differences in seagrass structure (density, length, biomass) and depth are the probable cause of the contrasting responses at the seaward and shoreward edges, but could not explain the difference between the middle and the seaward edge. 
Edges are a common feature of landscapes that influence environmental factors (Murcia 1995), species interactions (Fagan et al. 1999) and trophic transfer (Cadenasso et al. 2003). Seagrass habitats produce a distinct boundary with unvegetated sand habitats, producing changes in water flow (Bologna \& Heck 2002), sediment size (Fonseca \& Fisher 1986) and interactions between seagrass and sand predators and prey (Bologna \& Heck 1999). Seagrass edges are thought to represent a reflective or absorptive edge for seagrass specialists because of the lack of shelter outside seagrass patches. At both Sites 2 and 3, however, we found strong use of both the seaward seagrass and sand edges by fish, which may reflect a spillover effect, whereby aggregated individuals may 'spill' from a preferred habitat onto a non-preferred habitat (Ries \& Sisk 2004). Alternatively, the sand edge may provide a complementary habitat (Ries \& Sisk 2004), where water currents, food availability, foraging success and predation rates are beneficial.

Previous work on seagrass edge effects have produced inconsistent results (Connolly \& Hindell 2006). We found strong evidence of an edge effect in seagrass patches that could be both explained (shoreward edge) and not explained (seaward edge) by seagrass structure and depth. Fish used sand adjacent to seagrass extensively, further supporting the importance of seagrass edges to seagrass fish, but mechanisms establishing edge effects have only been hypothesised and need to be tested. As seagrass habitats are becoming increasingly fragmented around the world, there is an increased need to establish the effects and implications of edges to develop and improve the management of seagrass habitats.

Acknowledgements. We thank N. Gregor, J. Smith, T. O'Neil and S. Holt for field assistance; 3 anonymous reviewers for their comments, which improved the manuscript; the Victorian Marine Science Consortium for use of their facilities; and the Australian Research Council, which funded this project. All research was done in accordance with institutional, national and international guidelines concerning the use of animals in research.

\section{LITERATURE CITED}

Anderson T (2003) The functional relationship between temperate fishes and the associated seagrass landscapes. PhD thesis, The University of Melbourne

Bell SS, Brooks RA, Robbins BD, Fonseca MS, Hall MO (2001) Faunal response to fragmentation in seagrass habitats: implications for seagrass conservation. Biol Conserv 100: 115-123

Bologna PAX, Heck KL (1999) Differential predation and growth rates of bay scallops within seagrass habitats. J Exp Mar Biol Ecol 239:299-314

Bologna PAX, Heck KL (2002) Impact of habitat edges on density and secondary production of seagrass-associated fauna. Estuaries 25:1033-1044

Bostrom C, Jackson EL, Simenstad CA (2006) Seagrass landscapes and their effects on associated fauna: a review. Estuar Coast Shelf Sci 68:383-403

Cadenasso ML, Pickett STA, Weathers KC, Jones CG (2003) A framework for a theory of ecological boundries. BioScience 53:750-758

Connolly RM (1994) A comparison of fish assemblages from seagrass and unvegetated areas of a southern Australian estuary. Aust J Mar Freshw Res 45:1033-1044

> Connolly RM, Hindell JS (2006) Review of nekton patterns and ecological processes in seagrass landscapes. Estuar Coast Shelf Sci 68:433-444

- Edgar GJ, Shaw C (1995) The production and trophic ecology of shallow-water fish assemblages in southern Australia. 1. Species richness, size structure and production of fishes in Western Port, Victoria. J Exp Mar Biol Ecol 194:53-81

Fagan WE, Cantrell RS, Cosner C (1999) How habitat edges change species interactions. Am Nat 153:165-182

Fahrig L (2003) Effects of habitat fragmentation on biodiversity. Annu Rev Ecol Syst 34:487-515

$>$ Ferrell DJ, Bell JD (1991) Differences among assemblages of fish associated with Zostera capricorni and bare sand over a large spatial scale. Mar Ecol Prog Ser 72:15-24

Fonseca MS, Fisher JS (1986) A comparison of canopy friction and sediment movement between four species of seagrass with reference to their ecology and restoration. Mar Ecol Prog Ser 29:15-22

Gibson RN, Robb L, Burrows MT, Ansell AD (1996) Tidal, diel and longer term changes in the distribution of fishes on a Scottish sandy beach. Mar Ecol Prog Ser 130:1-17

Hindell JS, Jenkins GP, Keough MJ (2002) Variability in the numbers of post-settlement King George whiting (Sillaginidae: Sillaginodes punctata, Cuvier) in relation to predation, habitat complexity and artificial cage structure. J Exp Mar Biol Ecol 268:13-31

> Hovel KA, Fonseca MS, Myer DL, Kenworthy WJ, Whitfield PE (2002) Effects of seagrass landscape structure, structural complexity and hydrodynamic regime on macrofaunal densities in North Carolina seagrass beds. Mar Ecol Prog Ser 243:11-24

Hyndes GA, Kendrick AJ, MacArthur LD, Stewart E (2003) Differences in the species- and size-composition of fish assemblages in three distinct seagrass habitats with differing plant and meadow structure. Mar Biol 142:1195-1206

Jackson EL, Rowden AA, Attrill MJ, Bossey SJ, Jones MB (2001) The importance of seagrass beds as a habitat for fishery species. Oceanogr Mar Biol Annu Rev 39:269-303

> Jackson EL, Attrill MJ, Rowden AA, Jones MB (2006) Seagrass complexity hierarchies: influence on fish groups around the coast of Jersey (English Channel). J Exp Mar Biol Ecol 330:38-54

> Jelbart JE, Ross PM, Connolly RM (2006) Edge effects and patch size in seagrass landscapes: an experimental test using fish. Mar Ecol Prog Ser 319:93-102

> Jelbart JE, Ross PM, Connolly RM (2007) Patterns of small fish distributions in seagrass beds in a temperate Australian estuary. J Mar Biol Assoc UK 87:1297-1307

Jenkins GP, Hamer PA (2001) Spatial variation in the use of seagrass and unvegetated habitats by post-settlement King George whiting (Percoidei: Sillaginidae) in relation to meiofaunal distribution and macrophyte structure. Mar Ecol Prog Ser 224:219-229

> Kendrick AJ, Hyndes GA (2005) Variations in the dietary compositions of morphologically diverse syngnathid fishes. Environ Biol Fishes 72:415-427 
Murcia C (1995) Edge effects in fragmented forests: implications for conservation. Trends Ecol Evol 10:58-62

Nagelkerken I, Dorenbosch M, Verberk W, Cocheret de la Morinière E, van der Velde G (2000) Day-night shifts of fishes between shallow-water biotopes of a Caribbean bay, with emphasis on the nocturnal feeding of Haemulidae and Lutjanidae. Mar Ecol Prog Ser 194:55-64

Quinn GP, Keough MJ (2002) Experimental design and data analysis for biologists. Cambridge University Press

Ries L, Sisk TD (2004) A predictive model of edge effects. Ecology 85:2917-2926

Initial editorial responsibility: Howard Browman, Storebø,

Norway (until November 5, 2007); Final editorial responsibility:

Matthias Seaman, Oldendorf/Luhe, Germany
Sanchez-Jerez P, Barbera-Cebrian C, Ramos-Espla A (1999) Daily vertical migrations in the epifauna associated with Posidonia oceanica meadows. J Mar Biol Assoc UK 79: 971-977

Tanner JE (2005) Edge effects on fauna in fragmented seagrass meadows. Austral Ecol 30:210-218

Turner MG (1989) Landscape ecology: the effect of pattern on process. Annu Rev Ecol Syst 20:171-197

Uhrin AV, Holmquist JG (2003) Effects of propeller scarring on macrofaunal use of the seagrass Thalassia testudinum. Mar Ecol Prog Ser 250:61-70

Submitted: May 15, 2007; Accepted: November 21, 2007 Proofs received from author(s): April 16, 2008 